\title{
Two-Center Electron-Electron Correlation within the Independent Event Model
}

\author{
G. M. Sigaud and E. C. Montenegro \\ Departamento de Física, Pontifícia Universidade Católica do Rio de Janeiro, \\ Caixa Postal 38071, Rio de Janeiro, RJ 22452-970, Brazil
}

Received on 30 October, 2002.

\begin{abstract}
The Independent Event Model (IEVM) is used to analyze collision processes for systems with three and four electrons, in situations where up to three active electrons are involved and dynamical correlation effects play a major role. The model is applied to single electron loss and loss-ionization processes. All the possible ionization mechanisms for both collision partners in each exit channel are considered, including antiscreening, which, in the IEVM, can be taken into account in a consistent way along all the other ones, keeping unitarity. As a casestudy, the IEVM is applied to the analysis of the single loss of $\mathrm{He}^{+}, \mathrm{Li}^{+}$, and $\mathrm{Li}^{2+}$ ions by $\mathrm{He}$ atoms with the simultaneous single and double ionization of the target. We present plots of the cross sections of single electron loss accompanied or not by the ionization of the target as functions of the projectile energy. The calculations describe well the experimental energy dependence and the high-velocity absolute values for the cross sections.
\end{abstract}

\section{Introduction}

Collisions between dressed ions - considered here as ions which carry electrons - and neutral atoms, when both collision partners have active electrons, present a multiplicity of possible simultaneous exit channels which result in singleor multi-electron transitions within and between the participating systems. Some of these channels include the single or multiple ionization of the projectile and of the target atom, followed - or not - by the capture of one - or more - target electrons by the incoming ion. Particular attention, from both the experimental and theoretical points of view, has been drawn in recent years to the simultaneous ionization of dressed projectiles colliding with neutral noble gas targets, which is governed by two competing mechanisms, the so-called direct loss-ionization and antiscreening modes $[1,2,3,4]$. The importance of the dynamical electron correlation in this type of collision is now well established, in particular at intermediate and high velocities, where the electron-electron interaction (antiscreening) can dominate the total electron loss cross section $[5,6]$. This has been evinced experimentally by the measurement either of total cross sections [7, 8, 9] or of the momentum distributions of the recoil ions, which provide a definite signature for the antiscreening mechanism [10, 11, 12, 13].

The simultaneous occurrence of competing processes, mainly when they include two-center electron correlation, renders difficult a comprehensive theoretical description of the collision. Only in the simplest cases, single-channel analyses can be used to describe properly the experimental results. The pioneering works of Bates and Griffing [14] provided the first description of such electron-loss processes within the first-Born approximation. Actually, the behavior of both the screening and the antiscreening modes for light targets is conveniently described by first-order mod- els, such as the plane wave Born approximation (PWBA) $[2,3,15,16,17]$.

There are not many options available to treat collision systems with several potentially active electrons as a whole, mainly in collisions where the probabilities for several of the competing processes are of the same order of magnitude and, thus, have to be considered simultaneously, since they are no longer independent. If more than one electron undergo a transition, the description is obviously more complicated, since one needs to couple, in principle in an unitarized way, the probabilities of occurrence of each transition.

One class of useful models to describe situations like these includes those which are able to express many-electron probabilities in terms of single-electron ones. Among them, one of the most popular is the independent particle model (IPM) $[2,18]$. In the IPM, dynamical correlation effects due to the electron-electron interaction are explicitly neglected, and each electron is assumed to move in an effective singleor two-center potential. The probabilities for multiple transitions are then obtained by multiplying the transition probabilities for each independent electron, using, in the general case, the multinomial probability distribution to account for the statistics of the equivalent electrons [19]. Thus, the solution of the N-electron problem is reduced to solving the single-electron problem $\mathrm{N}$ times. Although the IPM may provide a relatively simple way of obtaining good estimates for complex systems, considerably simplifying the analysis of the collision dynamics, it presents two main limitations: (i) the transition probability for each channel is calculated using the same potential for all equivalent electrons, and (ii) it is not possible, within the IPM, to take into account systems where dynamical correlated effects are as important as uncorrelated ones. The (basic) assumption of independence between the electrons is, in many cases, not realistic, which often induces the inclusion of ad hoc modifications in 
the IPM, mostly in which concerns the dynamical electronelectron interaction. The fact that two-center electron correlation involves the interaction of electrons in each of the collision partners renders the imposition of the unitarity of the transition probabilties very difficult, because one needs to consider the collision system as a whole. If the condition of unitarity is only partially taken into account, the antiscreening can be included in the IPM [5, 6, 17, 20, 21, 22]. As long as the probabilities involved are small, this procedure provides feasible results. However, this approach cannot considered as completely satisfactory from the point of view of consistency.

In this paper, the independent event model (IEVM) $[23,24]$ is worked out to provide a detailed scheme in which one- and two-center electron transitions are treated in such a way that unitarity can be consistently imposed for the whole collision system. In the IEVM it is assumed that a given process is a succession of several independent events, during each of which one electron (or two, in the case of the antiscreening) is considered active, while the others remain passive. This means that each intermediate transition, which may lead to the final state, is treated independently. Thus, the electrons are not independent, in the sense that each intermediate transition probability is calculated using the potential of the actual system. This implies that one may have for a given process different probabilities for the same type of intermediate transition. The net probability of a process is, then, obtained by summing up the probabilities for all possible paths which lead to the final state, each path being considered as a chain of successive events, whose probability is the product of single-electron transition probabilities.

The IEVM has been used by several authors to describe processes occurring in collision systems with one or two active electrons [23, 24, 25, 26, 27, 28, 29, 30], although, to the authors' knowledge, not for processes which involve two-center electron-electron correlation. Both the IPM and the IEVM provide approximately the same probabilities for processes involving one or two active electrons; however, they present a fundamental difference, which becomes more important as the number of active electrons in the system increases.

The possibility of inclusion of dynamical electron correlation represents a strong conceptual advantage of the IEVM when compared to the IPM. Unlike the IPM, the IEVM retains the time ordering of the events [23, 24, 31]. Quantum mechanical treatments of time ordering and its close connection to dynamical electron correlation in ion-atom collisions are given by Stolterfoht [32], using a second-order semiclassical approximation, and McGuire et al. [33], by means of the Dyson time ordering operator. The very important reason for this advantage is that in the IEVM unitarity can be independently considered in each step, even when it involves two-center correlated electron-electron mechanisms, like the antiscreening. This happens because one can impose the unitarity of the probabilities of occurrence of all the events in a given step, irrespective of the fact that they involve one- or two-center transitions.

In a previous paper [34], the first attempts were presented of application of the IEVM to processes where up to three active electrons were involved. However, some inconsistencies were later found in the way the model was used at that instance, which will be discussed in detail later in the present paper. A summarized description of the modifications introduced to deal with those inconsistencies was presented in Ref. [35]. Here, the IEVM is developed in detail in order to be employed in the systematic analysis of the singleelectron loss of dressed projectiles by neutral targets, with the simultaneous single and double ionization of the target at the intermediate- to high-velocity regime. In section 2 , the main features of the IEVM are introduced and the recipes to obtain the total transition probabilities are fully presented. In section 3 , the calculations are compared with the cross sections of total single-electron loss and electron loss accompanied by single and double ionization of the target of $\mathrm{Li}^{+}$and $\mathrm{Li}^{2+}$ ions by $\mathrm{He}$ atoms $[36,37]$, and of $\mathrm{He}^{+}$ions also by He atoms $[38,39,40]$. The specific contributions of the antiscreening mechanism to the total cross sections are analyzed in section 4. Finally, some conclusions are drawn in section 5. The formulas obtained in the IEVM for the systems considered here are presented in the Appendix.

\section{The independent event model for two- and three-active electron sys- tems}

Let us consider a system with a total number $N$ of electrons and a net transition from an initial to a final state involving $n$ active electrons of the system. The essence of the IEVM is the following: each net transition can be described as the occurrence of $n$ events - where each of the active electrons undergoes a transition in each event - combined with $(N-n)$ non-events, where the other electrons of the system remain passive in the initial state. Thus, the probability describing each possible path to reach the final state will be composed of a product of $N$ terms, divided into $n$ probabilities of occurrence of events and $(N-n)$ probabilities of occurrence of nonevents (or, alternatively, the non-occurrence of an event). It should be remarked that this scheme allows the inclusion of an unitarization condition at each step.

Since the employment of the IEVM for systems with more than two electrons is not intuitive, we have chosen some specific collision systems and processes in order to exemplify its application and allow the comparison with available experimental data. They are the following:

(i) $\left(\mathrm{He}^{1+}, \mathrm{Li}^{1+, 2+}\right)+\mathrm{He}^{0} \rightarrow\left(\mathrm{He}^{2+}, \mathrm{Li}^{2+, 3+}\right)+\mathrm{He}^{0}$ : single loss with no target ionization;

(ii) $\left(\mathrm{He}^{1+}, \mathrm{Li}^{1+, 2+}\right)+\mathrm{He}^{0} \rightarrow\left(\mathrm{He}^{2+}, \mathrm{Li}^{2+, 3+}\right)+\mathrm{He}^{+}$: single-loss - single-ionization;

(iii) $\left(\mathrm{He}^{1+}, \mathrm{Li}^{1+, 2+}\right)+\mathrm{He}^{0} \rightarrow\left(\mathrm{He}^{2+}, \mathrm{Li}^{2+, 3+}\right)+\mathrm{He}^{2+}$ : single-loss - double-ionization.

In the equivalent cases of the $\mathrm{He}^{+}$and $\mathrm{Li}^{2+}$ projectiles, $N$ is 3 and $n$ is 1, 2 or 3 for situations (i), (ii) and (iii) above, respectively. This means that the total probability for case (i) will include 1 event (the ionization of $\mathrm{He}^{+}$or $\mathrm{Li}^{2+}$ ) and 2 non-events (the non-ionization of the $\mathrm{He}^{0}$ target), for case (ii) processes with 2 events (the ionization of $\mathrm{He}^{+}$or $\mathrm{Li}^{2+}$ and the single ionization of $\mathrm{He}^{0}$ ) and 1 non-event (the nonionization of the remaining $\mathrm{He}^{+}$target ion), and for case (iii) 3 events (the ionization of $\mathrm{He}^{+}$or $\mathrm{Li}^{2+}$ and the double ionization of $\left.\mathrm{He}^{0}\right)$.

For the electron loss of the $\mathrm{Li}^{+}$projectile, it is clear that the descriptions, within the IEVM, are rather more compli- 
cated than in the $\mathrm{He}^{+}$or $\mathrm{Li}^{2+}$ cases, since now $N$ is equal to 4 , while $n$ can still be 1,2 or 3 , which increases not only the alternative paths to reach a given final state, but also the number of terms in each product of single-electron transition probabilities which describe a possible path. Thus, one has to consider for case (i) above 1 event (the single ionization of $\mathrm{Li}^{+}$) and 3 non-events (the non-ionization of the remaining $\mathrm{Li}^{+}$projectile and of the $\mathrm{He}^{0}$ target), for case (ii) 2 events (the single ionization of $\mathrm{Li}^{+}$and $\mathrm{He}^{0}$ ) and 2 non-events (the non-ionization of the remaining $\mathrm{Li}^{+}$and $\mathrm{He}^{+}$), and for case (iii) 3 events (the single ionization of $\mathrm{Li}^{+}$and the double ionization of $\mathrm{He}^{0}$ ) and 1 non-event (the non-ionization of the remaining $\mathrm{Li}^{2+}$ ).

One important aspect that must be taken into account refers to the fact that, depending on the number of electrons in the system, there may be several equivalent paths to reach a given final state. This implies that a weighting factor has to be included to avoid the overcounting of the individual probabilities. The effect of the overcounting was not considered in Ref. [34] (see Refs. [35] and [41]). It has to be included in order to keep the overall consistency of the model. Just as an example, consider the loss of $\mathrm{Li}^{2+}$ with the He target remaining neutral, case (i) above and equation (A.4) of the Appendix. Thus, as mentioned before, there is one event (the ionization of $\mathrm{Li}^{2+}$ ) and two non-events (the non-ionization of the $\mathrm{He}^{0}$ target). However, these non-ionization events can occur following three different paths, corresponding to three different orderings in time: either the loss occurs in the first event and the last two events are the non-ionization of the He target by $\mathrm{Li}^{3+}$; or the first event is the non-ionization of $\mathrm{He}$ by the $\mathrm{Li}^{2+}$ projectile, the second event being the loss of $\mathrm{Li}^{2+}$ and the last one the non-ionization of the He target by $\mathrm{Li}^{3+}$; or else, the first two events are the non-ionization of $\mathrm{He}$ by the $\mathrm{Li}^{2+}$ projectile, the last one being the loss of $\mathrm{Li}^{2+}$. These three sequences are, in terms of the final states, obviously equivalent; thus, a weighting factor $1 / 3$ has to be included in the expression of the total probability, for this specific case. Of course this also happens with other transitions considered here, so that weighting factors appear in the corresponding equations for the total probabilities.

For collisions between low-Z partners at intermediate velocities, the probabilities for both electron loss and target ionization can be estimated from either first-order approximations or nonperturbative methods, and the electron capture channel may be neglected [2, 3, 17, 42]. Also, one must take into account the antiscreening mode, which contributes to the simultaneous projectile loss and target ionization $[2,15]$. Thus, the isolated transitions that will be considered here to contribute to the possible final states, with the related probabilities, are

$\left(\mathrm{P}^{i+}\right)+\left(\mathrm{T}^{q+}\right) \rightarrow\left(\mathrm{P}^{(i+1)+}\right)+\left(\mathrm{T}^{q+}\right):$ projectile loss (screening), with probability $\left(\mathcal{P}_{\mathrm{P}^{i+}}\right)_{\mathrm{T}^{q+}}$,
$\left(\mathrm{P}^{i+}\right)+\left(\mathrm{T}^{q+}\right) \rightarrow\left(\mathrm{P}^{i+}\right)+\left(\mathrm{T}^{(q+1)+}\right)$ : target ionization (screening), with probability $\left(\mathcal{P}_{\mathrm{T}^{q+}}\right)_{\mathrm{P}^{i+}}$, and

$\left(\mathrm{P}^{i+}\right)+\left(\mathrm{T}^{q+}\right) \rightarrow\left(\mathrm{P}^{(i+1)+}\right)+\left(\mathrm{T}^{(q+1)+}\right)$ : simultaneous projectile and target ionization (antiscreening), with probability $\left(\mathcal{P}_{\mathrm{A} \mathrm{P}^{i+} \mathrm{T}^{q+}}\right)$,

where $i$ and $q$ are the charge states of the projectile and the target, respectively, before each individual transition. In the notation employed here, $\left(\mathcal{P}_{\mathrm{A}}\right)_{\mathrm{B}}$ is the probability of a transition occurring in partner $\mathrm{A}$ induced by partner $\mathrm{B}$. In the case of the antiscreening this distinction is unnecessary because the transitions in both partners are correlated.

A very important point is that, except for the antiscreening, each single event involves the transition of one electron. In the antiscreening case, since it involves the ionization of two electrons, one in the target, the other in the projectile, it corresponds to a single two-electron event and not two single events. A single two-electron event is a single event in which two electrons are simultaneously excited due to the electron-electron dynamical correlation. Thus, the transition probabilities $\mathcal{P}$ are probabilities per event, corresponding to either single- or double-electron transitions. Here the main feature of the IEVM becomes clear: the fact that one- and two-center transitions are considered as events on the same foot, so that one can impose the unitarity of the probabilities of all the events in a given step, involving the transition of either one or two electrons, in a comprehensive and consistent way.

Using the prescription outlined above, one can obtain the total probabilities for electron loss of the $\mathrm{He}^{+}, \mathrm{Li}^{+}$and $\mathrm{Li}^{2+}$ projectiles for cases (i) to (iii) above. The corresponding expressions for these probabilities are presented in the Appendix.

In order to shorten the equations which provide the total probabilities, equations (A.1) through (A.9), the probabilities of non-occurrence of a given event, that is, the probability for a given combination of projectile and target states to remain as they are, with neither of them undergoing any transitions, have been introduced. These are denoted in those equations by $\left(\tilde{\mathcal{P}}_{\mathrm{P}^{i+} \mathrm{T}^{q+}}\right)$, representing the probability for the projectile and the target to remain in charge states $i$ and $q$, respectively. The expressions for these probabilities of non-occurrence are also shown in the Appendix.

The probabilities of non-occurrence of events, $\left(\tilde{\mathcal{P}}_{\mathrm{P}^{i+} \mathrm{T}^{q+}}\right)$, are directly related to the condition of unitarity which can be imposed at each step in the IEVM. Let us consider, for example, the probability $\left(\tilde{\mathcal{P}}_{\mathrm{L} i^{2+}} \mathrm{He}^{+}\right)$, given by equation (A.17), which represents the probability that the pair $\mathrm{Li}^{2+}-\mathrm{He}^{+}$remains unchanged after a given step. For the sake of comparison with the IPM, equation (A.17) is reproduced below,

$$
\begin{aligned}
\left(\tilde{\mathcal{P}}_{\mathrm{Li}^{2+} \mathrm{He}^{+}}\right)= & 1-\left(\mathcal{P}_{\mathrm{Li}^{2+}}\right)_{\mathrm{He}^{+}}-\left(\mathcal{P}_{\mathrm{He}^{+}}\right)_{\mathrm{Li}^{2+}}-\left(\mathcal{P}_{\mathrm{He}^{+}}\right)_{\mathrm{Li}^{2+}}\left(\mathcal{P}_{\mathrm{Li}^{2+}}\right)_{\mathrm{He}^{2+}} \\
& -\left(\mathcal{P}_{\mathrm{Li}^{2+}}\right)_{\mathrm{He}^{+}}\left(\mathcal{P}_{\mathrm{He}^{+}}\right)_{\mathrm{Li}^{3+}}-\left(\mathcal{P}_{\mathrm{ALi}^{2+}} \mathrm{He}^{+}\right),
\end{aligned}
$$

while the equivalent expression in the IPM is 


$$
\begin{aligned}
\left(\tilde{\mathcal{P}}_{\mathrm{L}^{2+} \mathrm{He}}\right)= & {\left[1-\left(\mathcal{P}_{\mathrm{Li}^{2+}}\right)_{\mathrm{H} e^{+}}\right]\left[1-\left(\mathcal{P}_{\mathrm{H} e^{+}}\right)_{\mathrm{L} i^{2+}}\right] } \\
& =1-\left(\mathcal{P}_{\mathrm{Li}^{2+}}\right)_{\mathrm{H} e^{+}}-\left(\mathcal{P}_{\mathrm{H} e^{+}}\right)_{\mathrm{L} i^{2+}}-\left(\mathcal{P}_{\mathrm{H} e^{+}}\right)_{\mathrm{L} i^{2+}}\left(\mathcal{P}_{\mathrm{Li}^{2+}}\right)_{\mathrm{H} e^{+}}
\end{aligned}
$$

The conceptual differences between the two approaches are evident. The IEVM considers the unitarization of the whole system in each event; as a consequence it includes not only the single and double one-center transitions with time ordering, but also the antiscreening. In the IPM, on the other hand, the unitarization is considered at each center: the first term in equation (2) is for the $\mathrm{Li}^{2+}$, while the second is for the $\mathrm{He}^{+}$. Thus, it does not include the antiscreening, nor the time ordering of the double one-center transition.

It should be noted that all the probabilities of nonoccurrence of events, Eqs. (A10) to (A21) of the Appendix, tend to 1 as the collision velocity increases, since the ionization probabilities decrease for high energies. Thus, for example, for high velocities Eq. (A5) becomes

$$
\begin{aligned}
\left(P_{23}^{01}\right)_{\mathrm{Li}} & \simeq\left(\mathcal{P}_{\mathrm{Li}^{2+}}\right)_{\mathrm{He}^{0}}\left(\mathcal{P}_{\mathrm{He}^{0}}\right)_{\mathrm{Li}^{3+}} \\
& +\left(\mathcal{P}_{\mathrm{He}^{0}}\right)_{\mathrm{Li}^{2+}}\left(\mathcal{P}_{\mathrm{Li}^{2+}}\right)_{\mathrm{He}^{+}}+\left(\mathcal{P}_{\mathrm{A} \mathrm{Li}^{2+} \mathrm{He}^{0}}\right)
\end{aligned}
$$

where $P_{23}^{01}$ (b) is the single-loss - single ionization probability for the $\mathrm{Li}^{2+}+\mathrm{He}^{+}$system. The first two terms in this equation represent the contribution due to the two-step nucleus-electron interaction, with time ordering, while the last term is the antiscreening mechanism.

In the IPM, the expression for this process is

$$
\left(P_{23}^{01}\right)_{\mathrm{Li}}=2\left(\mathcal{P}_{\mathrm{Li}^{2+}}\right)_{\mathrm{He}^{0}}\left(\mathcal{P}_{\mathrm{He}^{0}}\right)_{\mathrm{Li}^{2+}}+\left(\mathcal{P}_{\mathrm{A} \mathrm{Li}^{2+} \mathrm{He}^{0}}\right),
$$

However, in this expression the antiscreening is included in an ad hoc way, as mentioned before.

Comparison of these last two equations shows that in the IEVM the ordering in time of the events implies in explicitly considering different probabilities to achieve the same final state, even in the high-velocity regime, where the inclusion of the concept of non-occurrence of events may not be very relevant in numerical terms, but still assures the consistent inclusion of the antiscreening.

\section{Calculations of the probabilities and cross sections}

In this section we describe how the probabilities which appear in equations (A.1) to (A.21) were estimated, in order to make comparisons with experiment. The probabilities for ionization by charged particles were evaluated using the straight-line version of the semi-classical approximation presented by Hansteen et al. [43], with effective values for the charge of the projectile. In some cases, velocitydependent values of the effective charge were estimated through a Bohr-like potential, as indicated by Montenegro et al. [44]. The values obtained increased the ion charge state by factors ranging from 10 to $50 \%$ for energies between 0.5 and $16 \mathrm{MeV}$.

The probabilities for the projectile electron loss of $\mathrm{He}^{+}$, $\mathrm{Li}^{+}$and $\mathrm{Li}^{2+}$ by the neutral target atom $\mathrm{He}^{0}$ were calculated using the coupled-channel method $[17,45,46]$. In the calculations of all these probabilities, the effective atomic number and the ionization energies of the targets were taken from Refs. [47] and [48], respectively.

The antiscreening probabilities, as functions of the impact parameter, were estimated from cross sections calculated using the procedure introduced by Montenegro and Meyerhof within the PWBA [15]. Since the antiscreening is due to the overlap of the electron clouds of the projectile and the target, it ranges up to rather large values of the impact parameter of the collision. Thus, the antiscreening probabilities do not present a strong variation with the impact parameter and can be assumed to be constant, for simplicity. Calculations of the final cross sections assuming different "cut-off" values for the impact parameter showed differences smaller than $10 \%$ in all the calculated total cross sections. Thus, a "cut-off" impact parameter of 3.5 au was chosen, because, for this value, the ionization probabilities for all the other mechanisms considered here are already negligible in the velocity range of the experimental data.

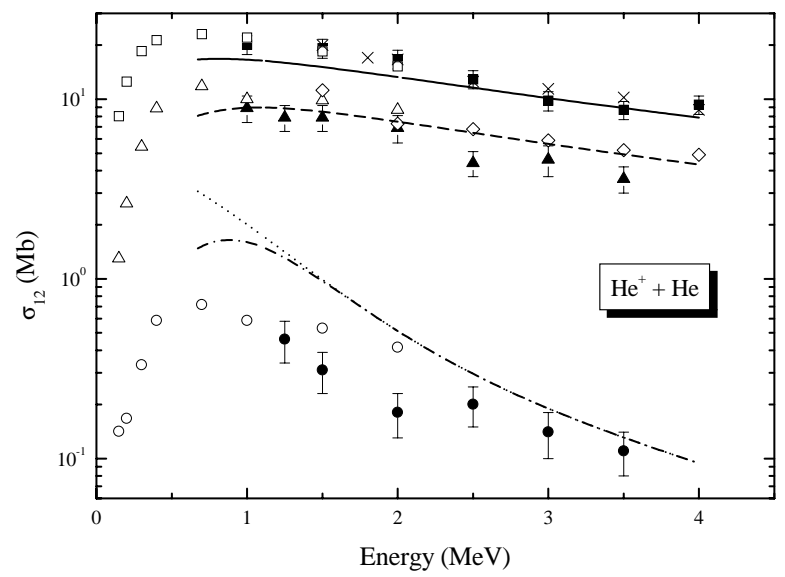

Figure 1. Electron loss cross sections of $\mathrm{He}^{+}$by neutral $\mathrm{He}$ as a function of the projectile energy. Experiment: open squares [39], crosses [40], and solid squares [38], total electron loss; open triangles [39], open diamonds [40], and solid triangles [38], single-loss - single-ionization; open circles [39]) and solid circles [38], singleloss - double-ionization. Theory: solid line, total electron loss (sum of Eqs. (A1) through (A3)); dashed line, single-loss - singleionization (Eq. (A2)); dotted line, single-loss - double-ionization (Eq. (A3)) without capture; dash-dotted line, single-loss - doubleionization (Eq. (A3)) with capture (see text).

In figure 1 the theoretical cross sections are compared with the experimental data for the electron loss of $\mathrm{He}^{+}$from DuBois [39], Montenegro et al. [40], and Santos [38] as a function of the projectile energy. In the figure, the trian- 
gles, the diamonds, and the dashed line are the results for the single-loss - single-ionization process (case (ii), above), while the circles and the dotted line are those for the singleloss - double-ionization process (case (iii), above). The squares, the crosses, and the solid line are the results for the total electron loss cross sections; the solid line corresponds, then, to the sum of the cross sections for all three processes given by equations (A.1) through (A.3).

For the electron loss of $\mathrm{Li}^{2+}$ by $\mathrm{He}$, the comparison with the experimental data of Woitke et al. [36] is shown in figure 2. In this figure, the triangles and the dotted line are the results for the single-loss - single-ionization process, while the circles and the dashed line are those for the single-loss double-ionization process. The squares are the experimental results for the total electron loss cross sections; thus, the solid line corresponds again to the sum of the cross sections for all three processes given by equations (A.4) to (A.6). In both cases, the calculations describe reasonably well the experimental energy dependence and the absolute values for the total loss and loss accompanied by target single ionization. In the case of the loss with double ionization of the target the agreement is good only in the high-velocity region.

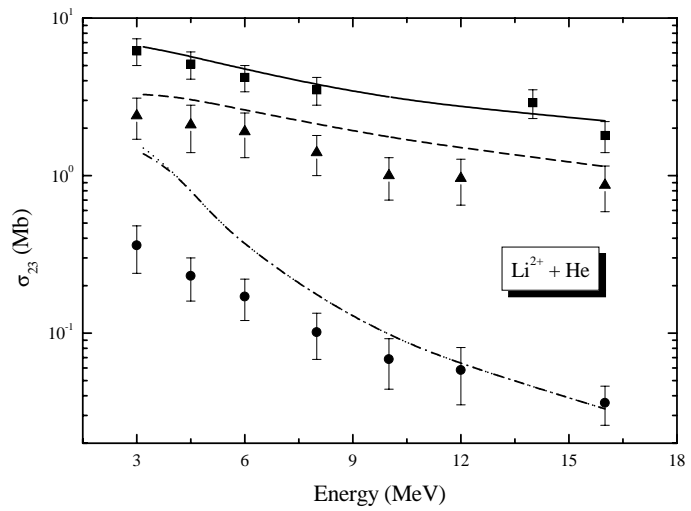

Figure 2. Same as in Fig. 1 for the $\mathrm{Li}^{2+}$ projectile. Experiment [36]: squares, total electron loss; triangles, single-loss - singleionization; circles, single-loss - double-ionization. Theory: solid line, total electron loss (sum of Eqs. (A4) through (A6)); dashed line, single-loss - single-ionization (Eq. (A5)); dotted line, singleloss - double-ionization (Eq. (A6)) without capture; dash-dotted line, single-loss - double-ionization (Eq. (A6)) with capture (see text).

For the lowest velocities, the lack of agreement is expected, due to the failure of the first-order models used to estimate the individual probabilities. In order to extend a little further the range of validity of the calculations using simple models, we used the procedure introduced by Sidorovich et al. [49] to circumvent probabilities greater than one, with the capture probabilities calculated as indicated by Ben-Itzhak et al. [50]. This procedure is already included in all the calculations presented here.

The comparison of the calculated cross sections with the experimental data of Woitke et al. [36] for the four-electron system $\mathrm{Li}^{+}+\mathrm{He}$ is shown in figure 3 . Here, the solid triangles and the dotted line are the results for the single-loss - single-ionization process, the solid circles and the dashed line correspond to the single-loss - double-ionization process, and the solid squares and the solid line are the results for the total electron loss cross sections. Also shown in the figure are the unpublished data from Shah [37] for the single projectile loss accompanied by the single (open triangles) and double (diamonds) target ionization. The agreement between the calculated and the experimental values is also good. However, deviations appear in all cases: for the total electron loss and single-loss - single-ionization processes they lie in the lowest velocity range, and for the single-loss - double-ionization the discrepancies actually occur in the energy dependence. Again, the capture probabilities were included in the procedure of Sidorovich et al. [49] for the low-velocity single-loss - double-ionization process.

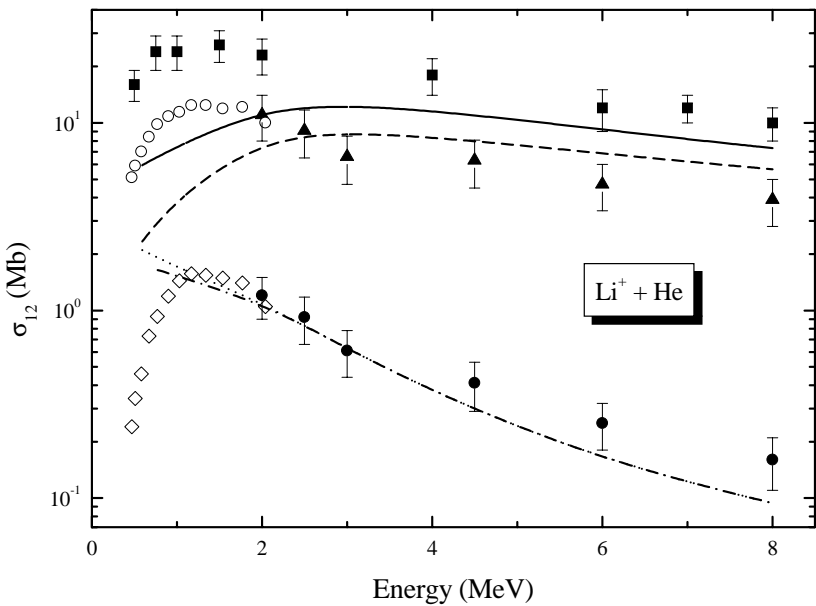

Figure 3. Same as in Fig. 1 for the $\mathrm{Li}^{+}$projectile. Experiment: solid squares, total electron loss [36]; solid triangles [36] and open circles [37], single-loss - single-ionization; solid circles [36] and open diamonds [37], single-loss - double-ionization. Theory: solid line, total electron loss (sum of Eqs. (A7) through (A9)); dashed line, single-loss - single-ionization (Eq. (A8)); dotted line, singleloss - double-ionization (Eq. (A9)) without capture; dash-dotted line, single-loss - double-ionization (Eq. (A9)) with capture (see text).

\section{Analysis of the antiscreening con- tribution}

The application of the IEVM in the way prescribed here allows the separation, in the total probability for the singleloss - single-ionization process, of the part containing explicitly the antiscreening probability and the factors that multiply it. From equations (A.2), (A.5), and (A.8) these terms are, respectively for the $\mathrm{He}^{+}, \mathrm{Li}^{2+}$ and $\mathrm{Li}^{+}$projectiles,

$$
\left.\left(P_{12}^{\text {anti }}\right)_{\mathrm{He}}=\frac{1}{2}\left(\mathcal{P}_{\mathrm{AHe}^{+} \mathrm{He}^{\mathrm{O}}}\right)\right]\left[\left(\tilde{\mathcal{P}}_{\mathrm{He}^{2+} \mathrm{He}^{+}}\right)+\left(\tilde{\mathcal{P}}_{\mathrm{He}^{+} \mathrm{He}^{0}}\right)\right],
$$




$$
\left.\left(P_{23}^{\text {anti }}\right)_{\mathrm{Li}}=\frac{1}{2}\left(\mathcal{P}_{\mathrm{A} \mathrm{Li}^{2+} \mathrm{He}^{0}}\right)\right]\left[\left(\tilde{\mathcal{P}}_{\mathrm{Li}^{3+} \mathrm{He}^{+}}\right)+\left(\tilde{\mathcal{P}}_{\mathrm{Li}^{2+} \mathrm{He}^{0}}\right)\right],
$$

and

$$
\left(P_{12}^{\text {anti }}\right)_{\mathrm{Li}}=\frac{1}{3}\left(\mathcal{P}_{\mathrm{A} \mathrm{Li}^{+} \mathrm{He}^{0}}\right)\left[\left(\tilde{\mathcal{P}}_{\mathrm{Li}^{2+} \mathrm{He}^{+}}\right)^{2}+\left(\tilde{\mathcal{P}}_{\mathrm{Li}^{+} \mathrm{He}^{0}}\right)\left(\tilde{\mathcal{P}}_{\mathrm{Li}^{2+} \mathrm{He}^{+}}\right)+\left(\tilde{\mathcal{P}}_{\mathrm{Li}^{+} \mathrm{He}^{0}}\right)^{2}\right],
$$

where $P_{i, i+1}^{\text {anti }}$ is the probability of electron loss associated directly to the antiscreening mechanism and the $\left(\tilde{\mathcal{P}}_{\mathrm{P}^{i+} \mathrm{T}^{q+}}\right)$ are the probabilities of non-occurrence of events, discussed in Section 2, and whose expressions are given in the Appendix.

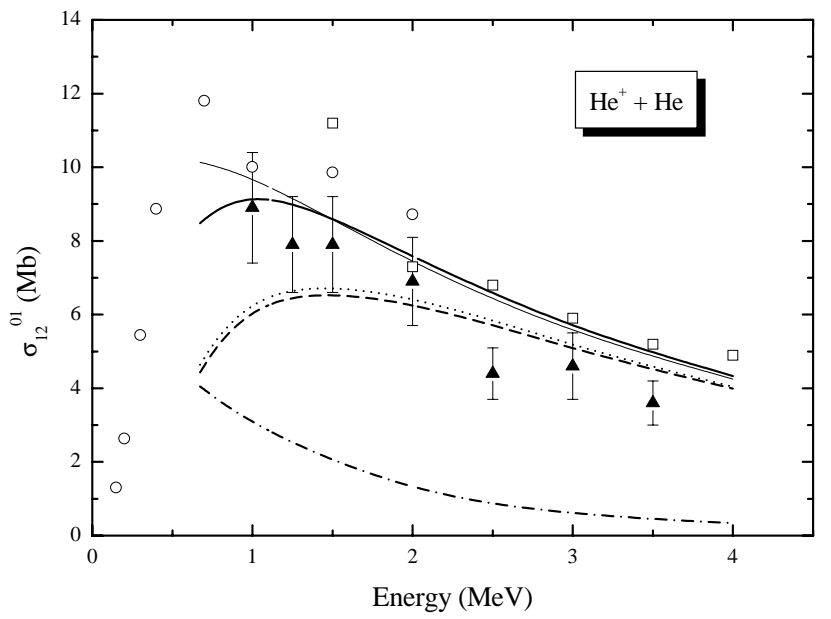

Figure 4. Single-loss - single-ionization cross sections for the $\mathrm{He}^{+}$ projectile as a function of the projectile energy. Experiment: open circles [39], open squares [40], and solid triangles [38]. Theory: thick solid line, total single-loss - single-ionization; dashed line, antiscreening contribution, this work; dotted line, antiscreening contribution calculated using the PWBA [15]; dash-dotted line, loss-ionization contribution (see text); thin solid line, total singleloss - single-ionization in the IPM.

These contributions were, then, integrated separately from the rest and compared to the corresponding experimental cross sections for the single-loss - single-ionization process. These comparisons are shown in figures 4 through 6 , for the $\mathrm{He}^{+}, \mathrm{Li}^{2+}$ and $\mathrm{Li}^{+}$projectiles, respectively. In these figures, the experimental data (solid triangles) are the same as in figures 1 to 3 , respectively, the thick solid lines are the calculated total single-loss - single-ionization cross sections, the dotted lines are the contributions from the antiscreening calculated using equations $(5)\left(\mathrm{He}^{+}\right),(6)\left(\mathrm{Li}^{2+}\right)$, and (7) $\left(\mathrm{Li}^{+}\right)$, and the dashed lines represent the antiscreening cross sections calculated according to Montenegro and Meyerhof within the PWBA [15]. Also included is the contribution of the direct loss-ionization (LI) process, i.e., the ionization of both partners due to the direct Coulomb nucleus-electron interaction (dash-dotted line), which was obtained by subtracting the antiscreening contribution from the total singleloss - single-ionization cross section. In figures 4 and 5, the total single-loss - single-ionization cross sections calculated within the IPM (equation (4) for the $\mathrm{Li}^{2+}$ and a similar one for the $\mathrm{He}^{+}$projectile) are also shown (thin solid lines).

It can be seen from these figures that the expected rapid decrease of the LI contribution as the energy increases is obtained [5]. Also, when the results for the two Li-ion projectiles are compared (figures 5 and 6), one sees that the existence of one more electron in the $\mathrm{Li}^{+}$makes the relative contribution of the LI process to the total cross section to be much smaller than for the $\mathrm{Li}^{2+}$ projectile, when compared to the antiscreening contribution. This happens because the second electron of the $\mathrm{Li}^{+}$projectile (i) increases the probability of occurrence of the antiscreening, and (ii) increases the screening of the $\mathrm{Li}^{+}$nuclear field, which decreases the probability for direct ionization for distant collisions.

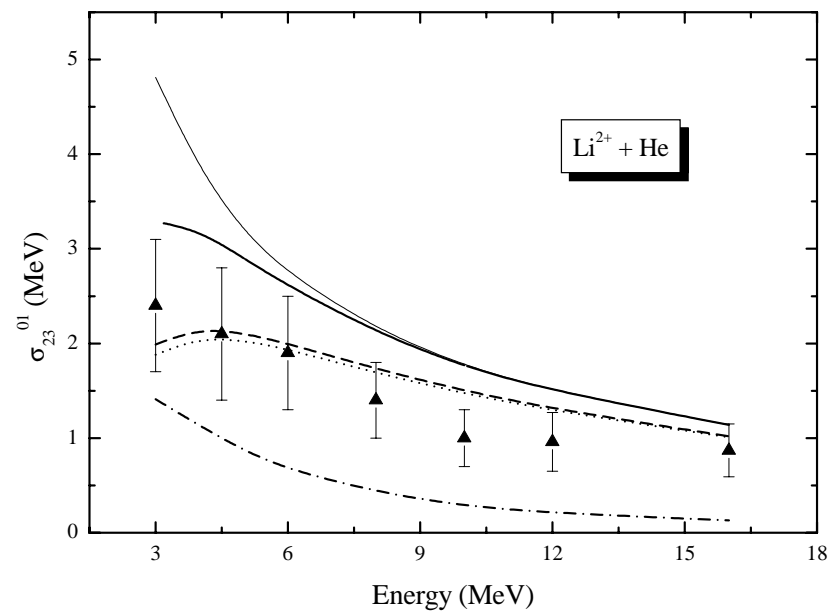

Figure 5. Same as in Fig. 4 for the $\mathrm{Li}^{2+}$ projectile. Experiment: solid triangles [36].

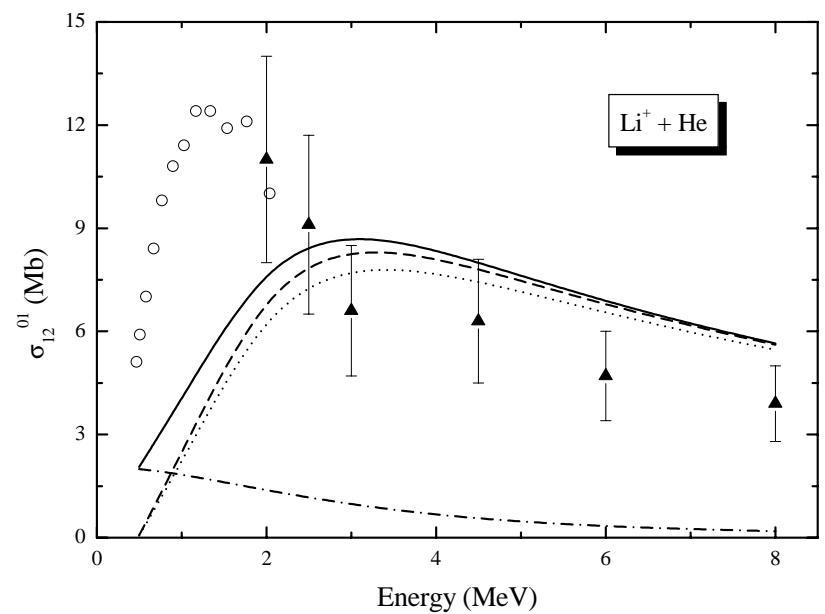

Figure 6. Same as in Fig. 4 for the $\mathrm{Li}^{+}$projectile. Experiment: solid triangles [36], open circles [37]. No calculation within the IPM is included in this figure.

Although most of the individual probabilities are calculated rather roughly, a comparison of the IEVM and the IPM 
predictions for the total single-loss - single-ionization cross sections is also presented in figures $4\left(\mathrm{He}^{+}\right)$and $5\left(\mathrm{Li}^{2+}\right)$ as an illustration of the numerical differences between these two models. At low energies, the differences between the IEVM and the IPM are significant, mainly for the $\mathrm{Li}^{2+}$ projectile. As discussed in the end of section 2, these differences arise from the combined effects of considering in the IEVM both the time ordering of the events - which implies in using ionization probabilities calculated with different charge states of the ionizing agents, as can be readily seen from the comparison between the first two terms of equation (3) and the first term of equation (4) - and the probabilities of non-occurrence of events. As the collision velocity increases, the antiscreening contribution dominates the total cross section, since the LI decreases very rapidly, as discussed above; thus, both models tend to coalesce.

\section{Conclusion}

The purpose of this paper was to include two-center correlated events in the independent event model in such a way that they could be taken into account consistently in the same framework as one-electron events in multi-electronic transitions. The present approach explores the important characteristic of the IEVM of keeping the unitarity of the whole system of colliding particles for each event.

As an illustration, the IEVM was applied to collision systems containing three and four electrons in processes in- volving up to three active electrons and in which the twocenter antiscreening mode can be as important as other onecenter ionization mechanisms. The calculations within this model were compared to experimental results of single electron loss of $\mathrm{He}^{+}, \mathrm{Li}^{+}$, and $\mathrm{Li}^{2+}$ by neutral He targets, accompanied - or not - by the single or double ionization of the target. This comparison showed a good overall agreement, even considering the fact that some probabilities were roughly estimated, which means that the IEVM can provide an useful tool for the understanding of multi-electronic processes in atomic collisions.

\section{Acknowledgments}

The authors wish to thank Profs. P. L. Grande for the coupled-channel calculations, M. B. Shah for the permission of using his unpublished data, and R. E. Olson for fruitful discussions. This work was supported in part by the Brazilian agencies CNPq, CAPES, FAPERJ, and MCT (PRONEX), and by the Volkswagen Stiftung (Germany).

\section{APPENDIX Expressions for the total probabilities}

The expressions for the total probabilities for electron loss, within the IEVM, of the $\mathrm{He}^{+}$projectile, $\left(P_{12}^{0 k}\right)_{\mathrm{He}}$, according to processes (i) to (iii) of Sec. 2, are the following

and

$$
\begin{aligned}
& \left(P_{12}^{00}\right)_{\mathrm{He}}=\frac{1}{3}\left(\mathcal{P}_{\mathrm{He}^{+}}\right)_{\mathrm{He}^{0}}\left[\left(\tilde{\mathcal{P}}_{\mathrm{He}^{2+} \mathrm{He}^{0}}\right)^{2}+\left(\tilde{\mathcal{P}}_{\mathrm{He}^{2+} \mathrm{He}^{0}}\right)\left(\tilde{\mathcal{P}}_{\mathrm{He}^{+} \mathrm{He}^{0}}\right)+\left(\tilde{\mathcal{P}}_{\mathrm{He}^{+} \mathrm{He}^{0}}\right)^{2}\right], \\
& \left(P_{12}^{01}\right)_{\mathrm{He}}=\frac{1}{3}\left\{\left(\mathcal{P}_{\mathrm{He}^{+}}\right)_{\mathrm{He}^{0}}\left(\mathcal{P}_{\mathrm{He}^{0}}\right)_{\mathrm{He}^{2+}}\left[\left(\tilde{\mathcal{P}}_{\mathrm{He}^{2+} \mathrm{He}^{+}}\right)+\left(\tilde{\mathcal{P}}_{\mathrm{He}^{2+} \mathrm{He}^{0}}\right)+\left(\tilde{\mathcal{P}}_{\mathrm{He}^{+} \mathrm{He}^{0}}\right)\right]\right. \\
& \left.+\left(\mathcal{P}_{\mathrm{He}^{0}}\right)_{\mathrm{He}^{+}}\left(\mathcal{P}_{\mathrm{He}^{+}}\right)_{\mathrm{He}^{+}}\left[\left(\tilde{\mathcal{P}}_{\mathrm{He}^{2+} \mathrm{He}^{+}}\right)+\left(\tilde{\mathcal{P}}_{\mathrm{He}^{+} \mathrm{He}^{+}}\right)+\left(\tilde{\mathcal{P}}_{\mathrm{He}^{+} \mathrm{He}^{0}}\right)\right]\right\} \\
& \left.+\frac{1}{2}\left(\mathcal{P}_{\mathrm{A} \mathrm{He}^{+} \mathrm{He}^{0}}\right)\right]\left[\left(\tilde{\mathcal{P}}_{\mathrm{He}^{2+} \mathrm{He}^{+}}\right)+\left(\tilde{\mathcal{P}}_{\mathrm{He}^{+} \mathrm{He}^{0}}\right)\right],
\end{aligned}
$$

$$
\begin{aligned}
\left(P_{12}^{02}\right)_{\mathrm{He}}= & \left(\mathcal{P}_{\mathrm{He}^{+}}\right)_{\mathrm{He}^{0}}\left(\mathcal{P}_{\mathrm{He}^{0}}\right)_{\mathrm{He}^{2+}}\left(\mathcal{P}_{\mathrm{He}^{+}}\right)_{\mathrm{He}^{2+}}+\left(\mathcal{P}_{\mathrm{A} \mathrm{He}^{+}+\mathrm{He}^{0}}\right)\left(\mathcal{P}_{\mathrm{He}^{+}}\right)_{\mathrm{He}^{2+}} \\
& +\left(\mathcal{P}_{\mathrm{He}^{0}}\right)_{\mathrm{He}^{+}}\left[2\left(\mathcal{P}_{\mathrm{He}^{+}}\right)_{\mathrm{He}^{+}}\left(\mathcal{P}_{\mathrm{He}^{+}}\right)_{\mathrm{He}^{2+}}+\left(\mathcal{P}_{\mathrm{A} \mathrm{He}^{+} \mathrm{He}^{+}}\right)\right] .
\end{aligned}
$$

Similar expressions hold for the total probabilities for electron loss of the $\mathrm{Li}^{2+}$ projectile, $\left(P_{23}^{0 k}\right)_{\mathrm{Li}}$, according to processes (i) to (iii) of Sec. 2. These are

$$
\begin{aligned}
\left(P_{23}^{00}\right)_{\mathrm{Li}}= & \frac{1}{3}\left(\mathcal{P}_{\mathrm{Li}^{2+}}\right)_{\mathrm{He}^{0}}\left[\left(\tilde{\mathcal{P}}_{\mathrm{Li}^{3+}} \mathrm{He}^{0}\right)^{2}+\left(\tilde{\mathcal{P}}_{\mathrm{Li}^{2+}} \mathrm{He}^{0}\right)\left(\tilde{\mathcal{P}}_{\mathrm{Li}^{3+} \mathrm{He}^{0}}\right)+\left(\tilde{\mathcal{P}}_{\mathrm{Li}^{2+} \mathrm{He}^{0}}\right)^{2}\right], \\
\left(P_{23}^{01}\right)_{\mathrm{Li}}= & \frac{1}{3}\left\{\left(\mathcal{P}_{\mathrm{Li}^{2+}}\right)_{\mathrm{He}^{0}}\left(\mathcal{P}_{\mathrm{He}^{0}}\right)_{\mathrm{Li}^{3+}}\left[\left(\tilde{\mathcal{P}}_{\mathrm{Li}^{3+}} \mathrm{He}^{+}\right)+\left(\tilde{\mathcal{P}}_{\mathrm{Li}^{3+} \mathrm{He}^{0}}\right)+\left(\tilde{\mathcal{P}}_{\mathrm{Li}^{2+}+\mathrm{He}^{0}}\right)\right]\right. \\
& \left.+\left(\mathcal{P}_{\mathrm{He}^{0}}\right)_{\mathrm{Li}^{2+}}\left(\mathcal{P}_{\mathrm{Li}^{2+}}\right)_{\mathrm{He}^{+}}\left[\left(\tilde{\mathcal{P}}_{\mathrm{Li}^{3+} \mathrm{He}^{+}}\right)+\left(\tilde{\mathcal{P}}_{\mathrm{Li}^{2+}} \mathrm{He}^{+}\right)+\left(\tilde{\mathcal{P}}_{\mathrm{Li}^{2+} \mathrm{He}^{0}}\right)\right]\right\} \\
& \left.+\frac{1}{2}\left(\mathcal{P}_{\mathrm{ALi}^{2+} \mathrm{He}^{0}}\right)\right]\left[\left(\tilde{\mathcal{P}}_{\mathrm{Li}^{3+} \mathrm{He}^{+}}\right)+\left(\tilde{\mathcal{P}}_{\mathrm{Li}^{2+}} \mathrm{He}^{0}\right)\right],
\end{aligned}
$$

and

$$
\begin{aligned}
\left(P_{23}^{02}\right)_{\mathrm{Li}}= & \left(\mathcal{P}_{\mathrm{Li}^{2+}}\right)_{\mathrm{He}^{0}}\left(\mathcal{P}_{\mathrm{He}^{0}}\right)_{\mathrm{Li}^{3+}}\left(\mathcal{P}_{\mathrm{He}^{+}}\right)_{\mathrm{Li}^{3+}}+\left(\mathcal{P}_{\mathrm{ALi}^{2+} \mathrm{He}^{0}}\right)\left(\mathcal{P}_{\mathrm{He}^{+}}\right)_{\mathrm{Li}^{3+}} \\
& +\left(\mathcal{P}_{\mathrm{He}^{0}}\right)_{\mathrm{Li}^{2+}}\left[\left(\mathcal{P}_{\mathrm{Li}^{2+}}\right)_{\mathrm{He}^{+}}\left(\mathcal{P}_{\mathrm{He}^{+}}\right)_{\mathrm{Li}^{3+}}+\left(\mathcal{P}_{\mathrm{He}^{+}}\right)_{\mathrm{Li}^{2+}}\left(\mathcal{P}_{\mathrm{Li}^{2+}}\right)_{\mathrm{He}^{2+}}\right. \\
& \left.+\left(\mathcal{P}_{\mathrm{A} \mathrm{Li}^{2+} \mathrm{He}^{+}}\right)\right] .
\end{aligned}
$$


The fact that the system $\mathrm{Li}^{+}+\mathrm{He}^{0}$ has 4 electrons and that the processes under consideration involve up to 3 active electrons makes the corresponding expressions for the probabilities, $\left(P_{12}^{0 k}\right)_{\mathrm{Li}}$, much more cumbersome than the equivalent ones (Eqs. (A1) to (A6)) for the $\mathrm{He}^{+}$and $\mathrm{Li}^{2+}$ projectiles, respectively. However, if one considers carefully all the possible paths that lead to the final states for cases (i) to (iii) of Sec. 2, they can be straightforwardly obtained. The final results are

$$
\begin{aligned}
& \left(P_{12}^{00}\right)_{\mathrm{Li}}=\frac{1}{4}\left(\mathcal{P}_{\mathrm{Li}^{+}}\right)_{\mathrm{He}^{0}}\left[\left(\tilde{\mathcal{P}}_{\mathrm{Li}^{2+} \mathrm{He}^{0}}\right)^{3}+\left(\tilde{\mathcal{P}}_{\mathrm{Li}^{2+} \mathrm{He}^{0}}\right)^{2}\left(\tilde{\mathcal{P}}_{\mathrm{Li}^{+} \mathrm{He}^{\mathrm{O}}}\right)\right. \\
& \left.+\left(\tilde{\mathcal{P}}_{\mathrm{Li}^{+} \mathrm{He}^{0}}\right)^{2}\left(\tilde{\mathcal{P}}_{\mathrm{Li}^{2+} \mathrm{He}^{\mathrm{O}}}\right)+\left(\tilde{\mathcal{P}}_{\mathrm{Li}^{+} \mathrm{He}^{0}}\right)^{3}\right] \\
& \left(P_{12}^{01}\right)_{\mathrm{Li}}=\frac{1}{6}\left\{( \mathcal { P } _ { \mathrm { Li } ^ { + } } ) _ { \mathrm { He } ^ { 0 } } ( \mathcal { P } _ { \mathrm { He } ^ { 0 } } ) _ { \mathrm { Li } ^ { 2 + } } \left[\left(\tilde{\mathcal{P}}_{\mathrm{Li}^{2+} \mathrm{He}^{+}}\right)^{2}+\left(\tilde{\mathcal{P}}_{\mathrm{Li}^{2+} \mathrm{He}^{0}}\right)\left(\tilde{\mathcal{P}}_{\mathrm{Li}^{2+} \mathrm{He}^{+}}\right)+\left(\tilde{\mathcal{P}}_{\mathrm{Li}^{2+}} \mathrm{He}^{0}\right)^{2}\right.\right. \\
& \left.+\left(\tilde{\mathcal{P}}_{\mathrm{Li}^{+} \mathrm{He}^{0}}\right)^{2}+\left(\tilde{\mathcal{P}}_{\mathrm{Li}^{+} \mathrm{He}^{0}}\right)\left(\tilde{\mathcal{P}}_{\mathrm{Li}^{2+} \mathrm{He}^{+}}\right)+\left(\tilde{\mathcal{P}}_{\mathrm{Li}^{+} \mathrm{He}^{0}}\right)\left(\tilde{\mathcal{P}}_{\mathrm{Li}^{2+} \mathrm{He}^{0}}\right)\right] \\
& +\left(\mathcal{P}_{\mathrm{He}^{0}}\right)_{\mathrm{Li}^{+}}\left(\mathcal{P}_{\mathrm{Li}^{+}}\right)_{\mathrm{He}^{+}}\left[\left(\tilde{\mathcal{P}}_{\mathrm{Li}^{2+} \mathrm{He}^{+}}\right)^{2}+\left(\tilde{\mathcal{P}}_{\mathrm{Li}^{2+} \mathrm{He}^{+}}\right)\left(\tilde{\mathcal{P}}_{\mathrm{Li}^{+} \mathrm{He}^{+}}\right)+\left(\tilde{\mathcal{P}}_{\mathrm{Li}^{+} \mathrm{He}^{+}}\right)^{2}\right. \\
& \left.\left.+\left(\tilde{\mathcal{P}}_{\mathrm{Li}^{+} \mathrm{He}^{0}}\right)^{2}+\left(\tilde{\mathcal{P}}_{\mathrm{Li}^{+} \mathrm{He}^{0}}\right)\left(\tilde{\mathcal{P}}_{\mathrm{Li}^{2+} \mathrm{He}^{+}}\right)+\left(\tilde{\mathcal{P}}_{\mathrm{Li}^{+}{ }^{+} \mathrm{He}^{0}}\right)\left(\tilde{\mathcal{P}}_{\mathrm{Li}^{+} \mathrm{He}^{+}}\right)\right]\right\} \\
& +\frac{1}{3}\left(\mathcal{P}_{\mathrm{ALi}^{+} \mathrm{He}^{0}}\right)\left[\left(\tilde{\mathcal{P}}_{\mathrm{Li}^{2+} \mathrm{He}^{+}}\right)^{2}+\left(\tilde{\mathcal{P}}_{\mathrm{Li}^{+} \mathrm{He}^{0}}\right)\left(\tilde{\mathcal{P}}_{\mathrm{Li}^{2+} \mathrm{He}^{+}}\right)+\left(\tilde{\mathcal{P}}_{\mathrm{Li}^{+} \mathrm{He}^{0}}\right)^{2}\right],
\end{aligned}
$$

and

$$
\begin{aligned}
& \left(P_{12}^{02}\right)_{\mathrm{Li}}=\frac{1}{4}\left\{\left(\mathcal{P}_{\mathrm{Li}^{+}}\right)_{\mathrm{He}^{0}}\left(\mathcal{P}_{\mathrm{He}^{0}}\right)_{\mathrm{Li}^{2+}}\left(\mathcal{P}_{\mathrm{He}^{+}}\right)_{\mathrm{Li}^{2+}}\left[\left(\tilde{\mathcal{P}}_{\mathrm{Li}^{2+} \mathrm{He}^{2+}}\right)+\left(\tilde{\mathcal{P}}_{\mathrm{Li}^{2+} \mathrm{He}^{+}}\right)+\left(\tilde{\mathcal{P}}_{\mathrm{Li}^{2+} \mathrm{He}^{0}}\right)+\left(\tilde{\mathcal{P}}_{\mathrm{Li}^{+} \mathrm{He}^{0}}\right)\right]\right. \\
& +\left(\mathcal{P}_{\mathrm{He}^{0}}\right)_{\mathrm{Li}^{+}}\left(\mathcal{P}_{\mathrm{He}^{+}}\right)_{\mathrm{Li}^{+}}\left(\mathcal{P}_{\mathrm{Li}^{+}}\right)_{\mathrm{He}^{2+}}\left[\left(\tilde{\mathcal{P}}_{\mathrm{Li}^{2+} \mathrm{He}^{2+}}\right)+\left(\tilde{\mathcal{P}}_{\mathrm{Li}^{+} \mathrm{He}^{2+}}\right)+\left(\tilde{\mathcal{P}}_{\mathrm{Li}^{+} \mathrm{He}^{+}}\right)+\left(\tilde{\mathcal{P}}_{\mathrm{Li}^{+} \mathrm{He}^{0}}\right)\right] \\
& +\left(\mathcal{P}_{\mathrm{He}^{0}}\right)_{\mathrm{Li}^{+}}\left(\mathcal{P}_{\mathrm{Li}^{+}}\right)_{\mathrm{He}^{+}}\left(\mathcal{P}_{\mathrm{He}^{+}}\right)_{\mathrm{Li}^{2+}}\left[\left(\tilde{\mathcal{P}}_{\mathrm{Li}^{2+}} \mathrm{He}^{2+}\right)+\left(\tilde{\mathcal{P}}_{\mathrm{Li}^{2+} \mathrm{He}^{+}}\right)+\left(\tilde{\mathcal{P}}_{\mathrm{Li}^{+} \mathrm{He}^{+}}\right)+\left(\tilde{\mathcal{P}}_{\mathrm{Li}^{+} \mathrm{He}^{0}}\right)\right] \\
& +\frac{1}{3}\left\{\left(\mathcal{P}_{\mathrm{He}^{0}}\right)_{\mathrm{Li}^{+}}\left(\mathcal{P}_{\mathrm{ALi}^{+} \mathrm{He}^{+}}\right)\left[\left(\tilde{\mathcal{P}}_{\mathrm{Li}^{2+} \mathrm{He}^{2+}}\right)+\left(\tilde{\mathcal{P}}_{\mathrm{Li}^{+} \mathrm{He}^{+}}\right)+\left(\tilde{\mathcal{P}}_{\mathrm{Li}^{+} \mathrm{He}^{0}}\right)\right]\right. \\
& \left.+\left(\mathcal{P}_{\mathrm{ALi}^{+} \mathrm{He}^{0}}\right)\left(\mathcal{P}_{\mathrm{He}^{+}}\right)_{\mathrm{Li}^{2+}}\left[\left(\tilde{\mathcal{P}}_{\mathrm{Li}^{2+} \mathrm{He}^{2+}}\right)+\left(\tilde{\mathcal{P}}_{\mathrm{Li}^{2+} \mathrm{He}^{+}}\right)+\left(\tilde{\mathcal{P}}_{\mathrm{Li}^{+} \mathrm{He}^{0}}\right)\right]\right\} \text {. }
\end{aligned}
$$

In all the equations above, the second digit in the superscripts, $k$, denotes the final state of the target.

The expressions for the probabilities of non-occurrence of a transition, that appear in Eqs. (A1) through (A3) for the $\mathrm{He}^{+}$ projectile, are the following

$$
\begin{gathered}
\left(\tilde{\mathcal{P}}_{\mathrm{He}^{2+} \mathrm{He}^{0}}\right)=1-\left(\mathcal{P}_{\mathrm{He}^{0}}\right)_{\mathrm{He}^{2+}}\left[1+\left(\mathcal{P}_{\mathrm{He}^{+}}\right)_{\mathrm{He}^{2+}}\right], \\
\left(\tilde{\mathcal{P}}_{\mathrm{He}^{+} \mathrm{He}^{0}}\right)=1-\left(\mathcal{P}_{\mathrm{He}^{+}}\right)_{\mathrm{He}^{0}}-\left(\mathcal{P}_{\mathrm{He}^{0}}\right)_{\mathrm{He}^{+}}-\left[1+\left(\mathcal{P}_{\mathrm{He}^{+}}\right)_{\mathrm{He}^{2+}}\right] \\
\times\left[\left(\mathcal{P}_{\mathrm{He}^{+}}\right)_{\mathrm{He}^{0}}\left(\mathcal{P}_{\mathrm{He}^{0}}\right)_{\mathrm{He}^{2+}}+2\left(\mathcal{P}_{\mathrm{He}^{0}}\right)_{\mathrm{He}^{+}}\left(\mathcal{P}_{\mathrm{He}^{+}}\right)_{\mathrm{He}^{+}}+\left(\mathcal{P}_{\mathrm{AHe}^{+} \mathrm{He}^{0}}\right)\right], \\
\left(\tilde{\mathcal{P}}_{\mathrm{He}^{2+} \mathrm{He}^{+}}\right)=1-\left(\mathcal{P}_{\mathrm{He}^{+}}\right)_{\mathrm{He}^{2+}},
\end{gathered}
$$

and

$$
\begin{aligned}
\left(\tilde{\mathcal{P}}_{\mathrm{He}^{+} \mathrm{He}^{+}}\right)= & 1-\left(\mathcal{P}_{\mathrm{He}^{+}}\right)_{\mathrm{He}^{+}}\left[2+\left(\mathcal{P}_{\mathrm{He}^{+}}\right)_{\mathrm{He}^{+}}+\left(\mathcal{P}_{\mathrm{He}^{+}}\right)_{\mathrm{He}^{2+}}\right] \\
& -\left(\mathcal{P}_{\mathrm{A} \mathrm{He}^{+} \mathrm{He}^{+}}\right) .
\end{aligned}
$$

For the $\mathrm{Li}^{+}$and $\mathrm{Li}^{2+}$ projectiles, the corresponding equations for the probabilities of non-occurrence of a transition used in Eqs. (A4) through (A9) are

$$
\begin{gathered}
\left(\tilde{\mathcal{P}}_{\mathrm{Li}^{3+} \mathrm{He}^{0}}\right)=1-\left(\mathcal{P}_{\mathrm{He}^{0}}\right)_{\mathrm{Li}^{3+}}\left[1+\left(\mathcal{P}_{\mathrm{He}^{+}}\right)_{\mathrm{Li}^{3+}}\right] \\
\left(\tilde{\mathcal{P}}_{\mathrm{Li}^{2+} \mathrm{He}^{0}}\right)=1-\left(\mathcal{P}_{\mathrm{Li}^{2+}}\right)_{\mathrm{He}^{0}}-\left(\mathcal{P}_{\mathrm{He}^{0}}\right)_{\mathrm{Li}^{2+}}-\left(\mathcal{P}_{\mathrm{He}^{0}}\right)_{\mathrm{Li}^{2+}}\left(\mathcal{P}_{\mathrm{He}^{+}}\right)_{\mathrm{Li}^{2+}}\left[1+\left(\mathcal{P}_{\mathrm{Li}^{2+}}\right)_{\mathrm{He}^{2+}}\right] \\
-\left[\left(\mathcal{P}_{\mathrm{Li}^{2+}}\right)_{\mathrm{He}^{0}}\left(\mathcal{P}_{\mathrm{He}^{0}}\right)_{\mathrm{Li}^{3+}}+\left(\mathcal{P}_{\mathrm{He}^{0}}\right)_{\mathrm{Li}^{2+}}\left(\mathcal{P}_{\mathrm{Li}^{2+}}\right)_{\mathrm{He}^{+}}+\left(\mathcal{P}_{\mathrm{A} \mathrm{Li}^{2+}} \mathrm{He}^{0}\right]\right] \\
\times\left[1+\left(\mathcal{P}_{\mathrm{He}^{+}}\right)_{\mathrm{Li}^{3+}}\right], \\
\left(\tilde{\mathcal{P}}_{\mathrm{Li}^{3+} \mathrm{He}^{+}}\right)=1-\left(\mathcal{P}_{\mathrm{He}^{+}}\right)_{\mathrm{Li}^{3+}},
\end{gathered}
$$




$$
\begin{aligned}
& \left(\tilde{\mathcal{P}}_{\mathrm{Li}^{2+}} \mathrm{He}^{+}\right)=1-\left(\mathcal{P}_{\mathrm{Li}^{2+}}\right)_{\mathrm{He}^{+}}-\left(\mathcal{P}_{\mathrm{He}^{+}}\right)_{\mathrm{Li}^{2+}}-\left(\mathcal{P}_{\mathrm{He}^{+}}\right)_{\mathrm{Li}^{2+}}\left(\mathcal{P}_{\mathrm{Li}^{2+}}\right)_{\mathrm{He}^{2+}} \\
& -\left(\mathcal{P}_{\mathrm{Li}^{2+}}\right)_{\mathrm{He}^{+}}\left(\mathcal{P}_{\mathrm{He}^{+}}\right)_{\mathrm{Li}^{3+}}-\left(\mathcal{P}_{\mathrm{ALi}^{2+} \mathrm{He}^{+}}\right), \\
& \left(\tilde{\mathcal{P}}_{\mathrm{Li}^{+} \mathrm{He}^{0}}\right)=1-\left(\mathcal{P}_{\mathrm{He}^{0}}\right)_{\mathrm{Li}^{+}}-\left(\mathcal{P}_{\mathrm{He}^{0}}\right)_{\mathrm{Li}^{+}}\left(\mathcal{P}_{\mathrm{He}^{+}}\right)_{\mathrm{Li}^{+}}-\left(\mathcal{P}_{\mathrm{Li}^{+}}\right)_{\mathrm{He}^{0}}-\left(\mathcal{P}_{\mathrm{Li}^{+}}\right)_{\mathrm{He}^{0}}\left(\mathcal{P}_{\mathrm{Li}^{2+}}\right)_{\mathrm{He}^{0}} \\
& -\left[\left(\mathcal{P}_{\mathrm{Li}^{+}}\right)_{\mathrm{He}^{0}}\left(\mathcal{P}_{\mathrm{He}^{0}}\right)_{\mathrm{Li}^{2+}}+\left(\mathcal{P}_{\mathrm{He}^{0}}\right)_{\mathrm{Li}^{+}}\left(\mathcal{P}_{\mathrm{Li}^{+}}\right)_{\mathrm{He}^{+}}+\left(\mathcal{P}_{\mathrm{ALi}^{+} \mathrm{He}^{0}}\right)\right] \\
& \times\left[1+\left(\mathcal{P}_{\mathrm{He}^{+}}\right)_{\mathrm{Li}^{2+}}+\left(\mathcal{P}_{\mathrm{Li}^{2+}}\right)_{\mathrm{He}^{+}}+\left(\mathcal{P}_{\mathrm{He}^{+}}\right)_{\mathrm{Li}^{2+}}\left(\mathcal{P}_{\mathrm{Li}^{2+}}\right)_{\mathrm{He}^{2+}}+\left(\mathcal{P}_{\mathrm{Li}^{2+}}\right)_{\mathrm{He}^{+}}\left(\mathcal{P}_{\mathrm{He}^{+}}\right)_{\mathrm{Li}^{3+}}\right] \\
& -\left(\mathcal{P}_{\mathrm{He}^{0}}\right)_{\mathrm{Li}^{+}}\left(\mathcal{P}_{\mathrm{He}^{+}}\right)_{\mathrm{Li}^{+}}\left(\mathcal{P}_{\mathrm{Li}^{+}}\right)_{\mathrm{He}^{2+}}\left[1+\left(\mathcal{P}_{\mathrm{Li}^{2+}}\right)_{\mathrm{He}^{2+}}\right] \\
& -\left(\mathcal{P}_{\mathrm{Li}^{+}}\right)_{\mathrm{He}^{0}}\left(\mathcal{P}_{\mathrm{Li}^{2+}}\right)_{\mathrm{He}^{0}}\left(\mathcal{P}_{\mathrm{He}^{0}}\right)_{\mathrm{Li}^{3+}}\left[1+\left(\mathcal{P}_{\mathrm{He}^{+}}\right)_{\mathrm{Li}^{3+}}\right] \text {, } \\
& \left(\tilde{\mathcal{P}}_{\mathrm{Li}^{+} \mathrm{He}^{+}}\right)=1-\left(\mathcal{P}_{\mathrm{Li}^{+}}\right)_{\mathrm{He}^{+}}-\left(\mathcal{P}_{\mathrm{He}^{+}}\right)_{\mathrm{Li}^{+}}-\left(\mathcal{P}_{\mathrm{Li}^{+}}\right)_{\mathrm{He}^{+}}\left(\mathcal{P}_{\mathrm{Li}^{2+}}\right)_{\mathrm{He}^{+}}\left[1+\left(\mathcal{P}_{\mathrm{He}^{+}}\right)_{\mathrm{Li}^{3+}}\right] \\
& -\left[\left(\mathcal{P}_{\mathrm{Li}^{+}}\right)_{\mathrm{He}^{+}}\left(\mathcal{P}_{\mathrm{He}^{+}}\right)_{\mathrm{Li}^{2+}}+\left(\mathcal{P}_{\mathrm{He}^{+}}\right)_{\mathrm{Li}^{+}}\left(\mathcal{P}_{\mathrm{Li}^{+}}\right)_{\mathrm{He}^{2+}}+\left(\mathcal{P}_{\mathrm{ALi}^{+} \mathrm{He}^{+}}\right)\right] \\
& \times\left[1+\left(\mathcal{P}_{\mathrm{Li}^{2+}}\right)_{\mathrm{He}^{2+}}\right], \\
& \left(\tilde{\mathcal{P}}_{\mathrm{Li}^{2+} \mathrm{He}^{2+}}\right)=1-\left(\mathcal{P}_{\mathrm{Li}^{2+}}\right)_{\mathrm{He}^{2+}},
\end{aligned}
$$

and

$$
\left(\tilde{\mathcal{P}}_{\mathrm{Li}^{+} \mathrm{He}^{2+}}\right)=1-\left(\mathcal{P}_{\mathrm{Li}^{+}}\right)_{\mathrm{He}^{2+}}\left[1+\left(\mathcal{P}_{\mathrm{Li}^{2+}}\right)_{\mathrm{He}^{2+}}\right] .
$$

\section{References}

[1] J. H. McGuire, N. Stolterfoht, and P. R. Simony, Phys. Rev. A 24, 97 (1981).

[2] E. C. Montenegro, W. E. Meyerhof, and J. H. McGuire, Adv. At. Mol. \& Opt. Phys. 34, 249 (1994).

[3] E. C. Montenegro, W. E. Meyerhof, J. H. McGuire, and C. L. Cocke, in The Physics of Electronic and Atomic Collisions - XIX International Conference, ed. L. J. Dubé, J. B. A. Mitchell, J. W. McConkey, and C. E. Brian (AIP Conference Proceedings 360, 515, AIP, New York (1995)).

[4] J. H. McGuire, Electron Correlation Dynamics in Atomic Collisions (Cambridge University Press, Cambridge, 1997), Ch. 8.

[5] E. C. Montenegro, G. M. Sigaud, and W. E. Meyerhof, Phys. Rev. A 45, 1575 (1992).

[6] E. C. Montenegro, A. C. F. Santos, W. S. Melo, M. M. Sant'Anna and G. M. Sigaud, Phys. Rev. Lett. 88, 013201 (2002).

[7] H. -P. Hülskötter, W. E. Meyerhof, E. Dillard, and N. Guardala, Phys. Rev. Lett. 63, 1938 (1989).

[8] H. -P. Hülskötter, B. Feinberg, W. E. Meyerhof, A. Belkacem, J. R. Alonso, L. Blumenfeld, E. A. Dillard, H. Gould, N. Guardala, G. F. Krebs, M. A. McMahan, M. E. RhoadesBrown, B. S. Rude, J. Schweppe, D. W. Spooner, K. Street, P. Thieberger, and H. E. Wegner, Phys. Rev. A 44, 1712 (1991).

[9] E. C. Montenegro, W. S. Melo, W. E. Meyerhof, and A. G. de Pinho, Phys. Rev. Lett. 69, 3033 (1992).

[10] R. Dörner, V. Mergel, R. Ali, U. Buck, C. L. Cocke, K. Froschauer, O. Jagutzki, S. Lencinas, W. E. Meyerhof, S. Nüttgens, R. E. Olson, H. Schmidt-Böcking, L. Spielberger,
K. Tökesi, J. Ullrich, M. Unverzagt, and W. Wu, Phys. Rev. Lett. 72, 3166 (1994).

[11] W. Wu, K. L. Wong, R. Ali, C. Y. Chen, C. L. Cocke, V. Frohne, J. P. Giese, M. Raphaelian, B. Walch, R. Dörner, V. Mergel, H. Schmidt-Böcking, and W. E. Meyerhof, Phys. Rev. Lett. 72, 3170 (1994).

[12] W. Wu, K. L. Wong, E. C. Montenegro, R. Ali, C. Y. Chen, C. L. Cocke, R. Dörner, V. Frohne, J. P. Giese, V. Mergel, W. E. Meyerhof, M. Raphaelian, H. Schmidt-Böcking, and B. Walch, Phys. Rev. A 55, 2771 (1997).

[13] H. Kollmus, R. Moshammer, R. E. Olson, S. Hagmann, M. Schulz, and J. Ullrich, Phys. Rev. Lett. 88, 103202 (2002).

[14] D. R. Bates and G. W. Griffing, Proc. Phys. Soc., London, Sect. A 66, 961 (1953); 67, 663 (1954); 68, 90 (1955).

[15] E. C. Montenegro and W. E. Meyerhof, Phys. Rev. A 43, 2289 (1991).

[16] E. C. Montenegro and T. J. M. Zouros, Phys. Rev. A 50, 3186 (1994).

[17] P. L. Grande, G. Schiwietz, G. M. Sigaud, and E. C. Montenegro, Phys. Rev. A 54, 2983 (1996).

[18] J. H. McGuire, Electron Correlation Dynamics in Atomic Collisions (Cambridge University Press, Cambridge, 1997), Ch. 4.

[19] M. M. Sant'Anna, E. C. Montenegro, and J. H. McGuire, Phys. Rev. A 58, 2148 (1998).

[20] W. S. Melo, M. M. Sant'Anna, A. C. F. Santos, G. M. Sigaud, and E. C. Montenegro, Phys. Rev. A 60, 1124 (1999).

[21] T. Kirchner, L. Gulyás, H. J. Lüdde, E. Engel, and R. M. Dreizler, Phys. Rev. A 58, 2063 (1998).

[22] T. Kirchner and M. Horbatsch, Phys. Rev. A 63, 062718 (2001). 
[23] D. S. F. Crothers and R. McCarroll, J. Phys. B: At. Mol. Phys. 20, 2835 (1987)

[24] K. M. Dunseath and D. S. F. Crothers, J. Phys. B: At. Mol. Opt. Phys. 24, 5003 (1991)

[25] R. E. Olson, J. Ullrich, and H. Schmidt-Böcking, J. Phys. B: At. Mol. Phys. 20, L809 (1987).

[26] R. E. Olson, J. Ullrich, and H. Schmidt-Böcking, Phys. Rev. A 39, 5572 (1989).

[27] D. P. Marshall, C. Le Sech, and D. S. F. Crothers, J. Phys. B: At. Mol. Opt. Phys. 26, L219 (1993).

[28] R. K. Janev, E. A. Solov'ev, and D. Jakimovski, J. Phys. B: At. Mol. Opt. Phys. 28, L615 (1995).

[29] L. A. Wehrman, A. L. Ford, and J. F. Reading, J. Phys. B: At. Mol. Opt. Phys. 29, 5831 (1996).

[30] A. L. Ford, L. A. Wehrman, K. A. Hall, and J. F. Reading, J. Phys. B: At. Mol. Opt. Phys. 30, 2889 (1997).

[31] N. Stolterfoht, Spectroscopy and Collisions of Few-Electron Ions, edited by M. Ivascu, V. Fluorescu, and V. Zoran (World Scientific, Singapore, 1989), p. 342.

[32] N. Stolterfoht, Phys. Rev. A 48, 2980 (1993).

[33] J. H. McGuire, A. L. Godunov, S. G. Tolmanov, Kh. Kh. Shakov, R. Dörner, H. Schmidt-Böcking, and R. M. Dreizler, Phys. Rev. A 63, 052706 (2001).

[34] G. M. Sigaud and E. C. Montenegro, Phys. Scr. T92, 420 (2001).

[35] G. M. Sigaud and E. C. Montenegro, in Photonic, Electronic and Atomic Collisions - XXII International Conference, ed. J. Burgdörfer, J. S. Cohen, S. Datz, and C. Randy Vane (Rinton Press, Princeton, 2002), pp 547-558.

[36] O. Woitke, P. A. Závodszky, S. M. Ferguson, J. H. Houck, and J. A. Tanis, Phys. Rev. A 57, 2692 (1998).
[37] M. B. Shah, private communication

[38] A. C. F. Santos, D.Sc. thesis, PUC-Rio, 1998; A. C. F. Santos, W. S. Melo, M. M. Sant'Anna, G. M. Sigaud, and E. C. Montenegro (to be published).

[39] R. D. DuBois, Phys. Rev. A 39, 4440 (1989).

[40] E. C. Montenegro, W. S. Melo, W. E. Meyerhof, and A. G. de Pinho, Phys. Rev. 48, 4259 (1993).

[41] Eqs. (1) and (2) of Ref. [34] are incorrect because the overcounting effect was not considered. The corresponding correct equations are Eqs. (A4) and (A5) of the present paper.

[42] A. B. Voitkiv, G. M. Sigaud, and E. C. Montenegro, Phys. Rev. A 59, 2794 (1999).

[43] J. M. Hansteen, O. M. Johnsen, and L. Kocbach, At. Data Nucl. Data Tables 15, 315 (1975).

[44] E. C. Montenegro, K. L. Wong, W. Wu, P. Richard, I. BenItzhak, C. L. Cocke, R. Moshammer, J. P. Giese, Y. D. Wang, and C. D. Lin, Phys. Rev. A 55, 2009 (1997).

[45] G. Schiwietz, Phys. Rev. A 42, 296 (1990).

[46] P. L. Grande, private communication.

[47] E. Clementi and C. Roetti, At. Data Nucl. Data Tables 14, 177 (1974); D. Cruz-Garritz, J. A. Chamizo, and A. Garritz, Estructura Atómica (Addison-Wesley Iberoamericana, Wilmington, 1991), Ch.8.

[48] CRC Handbook of Chemistry and Physics, $76^{\text {th }}$ ed., edited by David R. Lide (Chemical Rubber Company Press, Boca Raton, 1995), Ch. 10.

[49] V. A. Sidorovich, V. S. Nikolaev, and J. H. McGuire, Phys. Rev. A 31, 2193 (1985).

[50] I. Ben-Itzhak, A. Jain, and O. L. Weaver, J. Phys. B 26, 1711 (1993). 